University of Nebraska - Lincoln

DigitalCommons@University of Nebraska - Lincoln

Mechanical \& Materials Engineering Faculty

Publications

Mechanical \& Materials Engineering,

Department of

7-1999

Adaptive Sampling for Coordinate Metrology

Robert Edgeworth

Robert G. Wilhelm

Follow this and additional works at: https://digitalcommons.unl.edu/mechengfacpub

Part of the Mechanics of Materials Commons, Nanoscience and Nanotechnology Commons, Other Engineering Science and Materials Commons, and the Other Mechanical Engineering Commons

This Article is brought to you for free and open access by the Mechanical \& Materials Engineering, Department of at DigitalCommons@University of Nebraska - Lincoln. It has been accepted for inclusion in Mechanical \& Materials Engineering Faculty Publications by an authorized administrator of DigitalCommons@University of Nebraska Lincoln. 


\title{
Adaptive Sampling for Coordinate Metrology
}

\author{
Robert Edgeworth and Robert G. Wilhelm
}

Center for Precision Metrology, Department of Mechanical Engineering and Engineering Science, University of North Carolina at Charlotte, Charlotte, North Carolina, USA

Corresponding author - R. G. Wilhelm, email rgwilhel@uncc.edu

\begin{abstract}
An iterative sampling process for dimensional measurement is presented. The strategy is based upon the use of surface normal measurement data to develop an interpolating curve between sample points. The interpolant is used to select subsequent measurement targets iteratively. The process is repeated until the measurement converges to a complete and accurate evaluation of the surface. The required sample size is proportional to part quality. The most accurate parts will require the least sample points; whereas, lower quality parts will require a greater number of total samples. The method is particularly applicable to measurement of complex surfaces with coordinate measuring machines (CMMs).
\end{abstract}

Keywords: dimensional metrology, coordinate metrology, sampling, uncertainty

\section{Introduction}

Coordinate measuring machines (CMMs) are widely used in manufacturing to verify and control the dimensional accuracy of manufactured goods. Through a variety of mechanisms, these machines determine the position of points on the surface of the object under inspection. After a set of point measurements has been collected, part conformance to the design intent can be evaluated. Typically, groups of points are associated with nominal feature shapes and fitted to the geometry. The fit parameters are then compared to the design intent to determine conformance. The validity of this evaluation process is dependent upon the assumption that the set of sample points is representative of the surface being inspected. Current practice is to develop a sampling process that is constant for a given 
part design [1-5]. This static sampling process will perform acceptably for the majority of actual part shapes, but leaves open the possibility of interaction between the sampling plan and part errors. We have shown previously that the interaction between sampling patterns and part errors can result in fitting errors equal in magnitude to the part form error [6]. This paper will illustrate an iterative sampling method that determines a sampling pattern for individual parts based upon the use of surface normal measurements to predict the part errors between sample points.

CMMs capable of measuring surface normals have been available for many years. The primary application of this capability has been to maintain contact with the part while the probe traces a line across the part surface (scanning). This type of measurement permits a much higher data collection rate than the standard point-to-point probing technique used by most CMMs. However, scanning requires a machine with controls and dynamic stiffness of a much higher caliber than a point-to-point probing CMM. The result is that CMMs capable of scanning are typically priced from two to five times higher than a point probing machine with a similar work volume. The cost of probe heads capable of generating surface normal measurements represents only a small percentage of the price differential between scanning and point probing CMMs. This work presumes the availability of an intermediate product between the point probing and scanning machine classes; that is, a point probing $\mathrm{CMM}$ that also generates surface normal measurements. Sensors suitable for this type of machine have been demonstrated by a number of researchers recently [7-10].

The method uses the fact that the deviation in the measured surface normal indicates the rate of change in the surface error at the measurement point to develop an estimate of the errors between measurement points. Using this error estimate, we iteratively select regions where the predicted errors exceed our measurement uncertainty criteria and resample these areas until the error surface estimate converges to our measuring criteria. In this paper, we develop the interpolation model and show examples of the algorithm performance for two-dimensional (2-D) sections.

This sampling strategy is particularly relevant using min-max fitting algorithms. These fitting algorithms, typically applied to meet the intent of the ANSI Y14.5 tolerance standard, are constrained by the extreme points of the dataset under analysis. This dependence on extrema results in min-max algorithms being highly sensitive to the measurement points available in the sample set [11-16]. Our algorithm ensures that extreme points that do not represent surface discontinuities will be represented in the dataset, within a known uncertainty range.

\section{Optimization issues for sampling in coordinate metrology}

Three principal factors must be addressed to optimize a sampling plan for a dimensional measurement. The first factor to consider is how the sampling plan will affect the total measurement time, which directly correlates to the cost of the measurement. The second issue is the accuracy of any design tolerance evaluation performed using the sample points. The final, and often overlooked, issue is the completeness of the measurement. 
A CMM requires significant capital investment, both for the equipment itself and for the infrastructure required to maintain suitable environmental conditions for proper operation. Skilled operators are generally required to oversee the measurements and interpret measurement results. It is desirable, therefore, to maximize the output of these machines by developing a measurement algorithm that produces valid results in minimum time. Because total measurement time is proportional to the number of sample points required for the measurement cycle, minimizing the number of sample points collected is an important factor to consider when developing an optimal measurement plan.

Hocken et al. [11], show that using minimal sample sets to develop substitute geometry representations of part features can lead to poor measurement accuracy when form errors are present. They note that current industry practice is to sample far fewer points than are necessary to ensure development of accurate substitute features. Tolerances evaluated using Chebyshev algorithms are particularly sensitive to the sample set provided to the calculation [12-15]. An inaccurate measurement can lead to lost production, scrapped materials and, in extreme cases, the loss of a customer. These costs can be many orders of magnitude higher than the cost of the measurement itself. An accurate and reliable measurement result is an important factor to consider when developing a sampling plan.

An accurate measurement result does not ensure that the evaluation of the feature is complete. A complete measurement contains a full evaluation of the errors present on the part. In contrast, an accurate measurement requires only points representing the mean shape of the feature and the two extreme points above and below the mean line. Summerhays et al. [1], have demonstrated methods for generating accurate, but incomplete, sampling plans for cylinders based upon process knowledge of the expected modes for the complete error shape. A complete error assessment is of particular importance when measurement results are used for automated process feedback [17] [18]. Complete error evaluations can also reduce the time required to diagnose and correct manufacturing errors when process problems appear and human intervention is required.

Weckenmann et al. [19] suggest that a good sampling strategy will lead to:

1. the parameters of the substitute feature sought, to be correctly determined from the data points sensed

2. in a minimum time

3. using a suitably evaluation criterion

4. with sufficient reliability

5. and within a predetermined confidence range.

In addition, for a sampling solution to be generally applied, the methodology should be independent of the nominal feature shape, manufacturing process, and make no assumptions about the distribution of errors across the surface.

Current research indicates that a general solution to the sampling problem is not possible with established measurement practices. We attempt to solve the problem by using the increased information content available when both the surface normal and the position in space of surface points are measured. Using the deviations of location and surface normal 
from nominal, we have an indication of how the form error changes locally. This information is used to develop an interpolating curve that predicts the form error between pairs of measurement points. The curve is then used to decide if subsequent measurement points are required to complete the measurement within desired confidence limits and, if so, the optimal locations for further sample.

\section{Error surface model}

\subsection{Interpolation between measurement points}

Interpolation between measurement points is a widely used practice today. Graphic presentation of surface finish, roundness, and surface profiling all use linear interpolation to fill in the gaps between measurement points. Because these applications measure position only, a linear model is the sole possible solution between pairs of points. The interpolation is performed by first estimating the perpendicular deviation of each sample point from the nominal surface. Then lines are computed between each pair of points. The dependent axis is the surface error (e) and the independent axis is the distance along the surface (s). This coordinate system is the error space. Typically, the line segments in error space are magnified and transformed back to the part coordinate system to present the inspection results graphically. When measuring position data, the distinction between the error space and part coordinate system is largely trivial, and rarely is it developed formally. When dealing with position and normal vector measurements, this distinction is more important and greatly simplifies development of our ideas. Transformation of measurement data into error space makes the data independent of the nominal geometry and permits use of a consistent methodology, regardless of part complexity. Figure 1 shows nominal and actual position and surface normals for two sample shapes transformed into error space.
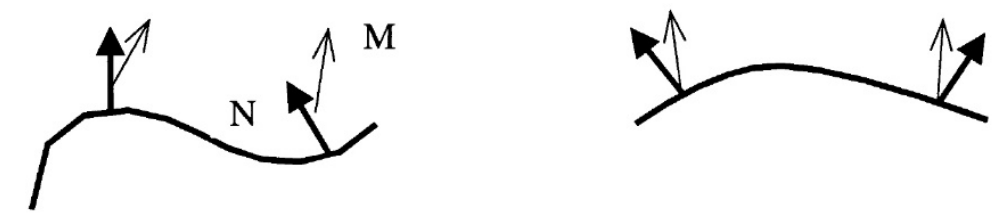

$\varepsilon$

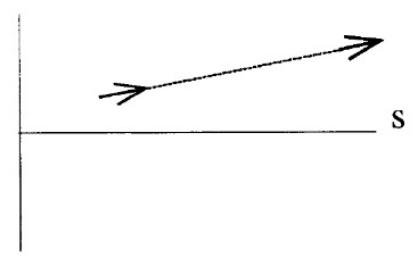

$\varepsilon$

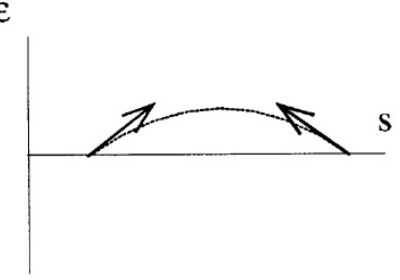

Figure 1. Transformation of measured position and surface normals into error space; interpolated errors are shown as a dotted curve. 
The bold lines in the top half of the figure represent a nominal shape with nominal surface normals shown at two locations. The lighter arrows represent actual measurements, with the base of the arrow representing the surface position measured, and the arrow direction indicating the measured surface normal. The bottom half of the figure shows the same data after transformation into error space. The arrows represent the position and surface normal deviation from nominal, and the dotted lines suggest an interpolant between the pair of sample points. Transformation of surface normals into error space and computation of the interpolant is developed below.

\subsection{Transformation of 2-D position and surface normal vectors into error space}

We want to transform both measurements of position and surface normal vectors into error space. The position deviation is defined as the minimum perpendicular distance from the measured point to the surface. Note that the direction of the perpendicular will be the nominal surface normal. We have effectively reduced the dimensionality of the position data from a vector to the scalar e. We want to perform the same dimension reduction for the surface normal measurement.

Consider a general, continuous curve $C$. The vector $N$ represents the nominal unit normal vector for the curve at a given point $P$ [Eq. (1)]. This vector can be computed in terms of $\operatorname{arc}$ distance $(s)$ as:

$$
\frac{\partial C}{\partial s}
$$

The true surface tangent at the sample point is the sum of the nominal tangent and the tangent of the manufacturing error. The surface normal we measure is perpendicular to the tangent [Eq. (2)] and can be expressed as:

$$
\frac{\partial C_{\text {actual }}}{\partial s}
$$

which is the normalized vector of the sum of vectors $N$ and $E$. Figure 2 is a sketch of the relation between $N, E$, and $M$. The measured direction is correct, but it is not possible to determine the magnitude $|N+E|$.

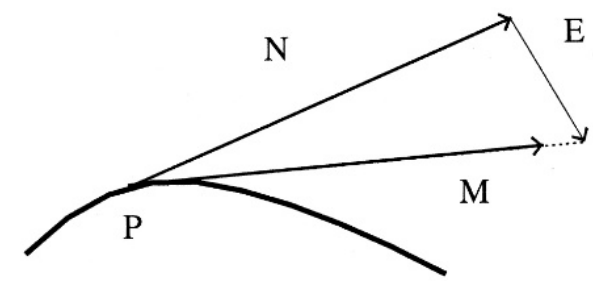

Figure 2. Relationship between nominal tangent vector $(\mathrm{N})$, tangent error vector $(\mathrm{E})$, and measured tangent $(\mathrm{M})$. 
The information that is lost because of the normalization of the vector $M$ represents the error in the rate that the surface is being developed. For example, if $|N+E|>1$, the curve in the area of the sample point is being traversed "too quickly," and the resulting curve will be shorter than intended. Similarly, if $|N+E|<1$, then the curve is being developed more slowly than was intended, and the arc length of the curve will be longer than expected in the area being sampled. If either error is systematic, subsequent sample points will detect the error, so the loss of information, although undesirable, will not render evaluation of the surface using normal vectors unworkable.

The surface normal error vector is

$$
\epsilon^{\prime}=N-M
$$

For the case of a 2-D curve with small errors in the surface normal vectors, we can approximate the error as a scalar quantity

$$
\epsilon^{\prime}=\sin \theta
$$

where $\theta$ is the angle between the nominal and measured surface normal vectors. The symbol $\epsilon^{\prime}$ is chosen because it describes the rate of change of the surface error e with respect to distance along the surface

$$
\epsilon^{\prime}=(\delta \epsilon / \delta s)
$$

We now have four parameters in error space for each pair of measurement points on our surface. We can interpolate the surface error between measurement points as a cubic polynomial

$$
\epsilon(u)=a_{0}+a_{1} u+a_{2} u^{2}+a_{3} u^{3}
$$

The inputs for this polynomial are the positions and the derivatives at the endpoints, which corresponds exactly to the Hermite form of a cubic polynomial. A sample calculation of the polynomial coefficients is given in the Appendix. By interpolating a cubic polynomial between pairs of measurement points, we develop a cubic spline representation of the surface errors. The resulting error estimating curve exhibits $\mathrm{C}^{1}$ continuity.

Spline interpolations exhibit several important advantages over polynomial interpolation, most importantly the variation diminishing property [20]. This property means that the interpolation surface will not exhibit more intersections with any given plane than the spline's control polygon. Therefore, the interpolation method described will not produce an error surface with more waves than the true surface. The interpolation model will also converge with the true error surface as sample size increases. However, it does not constrain the interpolated surface to be bound by the true error surface, so it is possible that the error interpolation might exaggerate the surface errors between sample points. 


\section{Uncertainty of the interpolating curve}

To obtain an estimate of the accuracy and reliability of the interpolant, the first issue to consider is variation in the curve attributable to the uncertainty in measurement of the position and surface normal at each point. We also consider how the actual wavelength of the error between measurement points will affect our interpolant.

The interpolating curve developed between two measurement points can be expected to vary as a result of the uncertainty of measurement of both the position and surface normal measurement. The cubic equation describing the curve can be split into two components; the ideal cubic interpolant, and the curve attributable to measurement error. The measuring error curve is also a cubic polynomial. The actual interpolating curve is the sum of these two components:

$$
\mathrm{G}_{\mathrm{a}}=\mathrm{G}_{\mathrm{i}}+\mathrm{Ge}_{\mathrm{e}}
$$

The ideal interpolant is not an exact representation of the error surface between measurement points. It is merely the interpolating cubic polynomial that would be found using the true values of position and surface normal at the sample points. Consider the cubic error function Ge. The function can be written in the Hermite form as

$$
\mathrm{Ge}(\mathrm{u})=\mathrm{b}_{3} \mathrm{u}^{3}+\mathrm{b}_{2} \mathrm{u}^{2}+\mathrm{b}_{1} \mathrm{u}+\mathrm{b}_{0} \quad 0 \leq \mathrm{u} \leq 1
$$

If the position and surface normal measurement errors were known at each point, the cubic coefficients could be found from the following:

$$
\begin{aligned}
& \mathrm{b}_{3}=2 \mathrm{Pe}_{\mathrm{e}}(0)-2 \mathrm{Pe}_{\mathrm{e}}(1)+\mathrm{dNe}_{\mathrm{e}}(0)+\mathrm{dN}_{\mathrm{e}}(1) \\
& \mathrm{b}_{2}=-3 \mathrm{Pe}(0)+3 \mathrm{Pe}(1)-2 \mathrm{dNe}_{\mathrm{e}}(0)-\mathrm{dNe}(1) \\
& \mathrm{b}_{1}=\mathrm{Ne}_{\mathrm{e}}(0) \\
& \mathrm{b}_{0}=\mathrm{Pe}_{\mathrm{e}}(0)
\end{aligned}
$$

$\mathrm{Pe}_{\mathrm{e}}$ is the position measurement error, and $\mathrm{N}_{\mathrm{e}}$ is the error in the measurement of surface normal. Because of the parameterization of the Hermite form from 0 to $1, \mathrm{Ne}$ must be scaled by $d$, the distance between sample points.

Five independent parameters control the shape of the uncertainty curve. We want to find the bounding shape that encloses all of the possible error curves. The four measurement uncertainty terms could combine in $64\left(4^{3}\right)$ ways to produce a maximum curve. Also, the distance term changes the magnitude of the error curve in a nonlinear fashion. To simplify evaluation of this function, we separate the error polynomial into the sum of two functions dependent only upon the surface normal or position measurement uncertainty. 
To solve for the first independent function, we assume that the surface normal measurement errors are zero. This is the case for measurements using only position data. In such cases, the error function for the position terms can be written as a line between the two sample points. Using the parameters shown above:

$$
\begin{aligned}
& \mathrm{b}_{0}=\mathrm{Pe}_{\mathrm{e}}(0) \\
& \mathrm{b}_{1}=\mathrm{Pe}_{\mathrm{e}}(1)-\mathrm{Pe}_{\mathrm{e}}(0) \\
& \mathrm{b}_{2}=0 \\
& \mathrm{~b}_{3}=0
\end{aligned}
$$

Setting the position measurement error terms to zero, we can find the uncertainty function for the surface normal measurements:

$$
\begin{aligned}
& \mathrm{b}_{3}=\mathrm{Ne}_{\mathrm{e}}(0)+\mathrm{Ne}_{\mathrm{e}}(1) \\
& \mathrm{b}_{2}=-2 \mathrm{Ne}(0)-\mathrm{Ne}(1) \\
& \mathrm{b}_{1}=\mathrm{Ne}_{\mathrm{e}}(0) \\
& \mathrm{b}_{0}=0
\end{aligned}
$$

Summing these two functions and subtracting from the original Hermite function gives the deviation from an exact solution that results from our separation of the error terms:

$$
\text { Separation Error }=(\mathrm{Pe}(1)-\mathrm{Pe}(0))\left(-2 \mathrm{u}_{3}+3 \mathrm{u}_{2}-\mathrm{u}\right)
$$

The deviation is a function of the difference between the position measurement errors at each sample point only. The maximum deviation from the exact form is 0.096 times the difference between the position measurement errors at each sample point. Therefore, using the simplified model will give a result that is within $10 \%$ of the true measurement error. More importantly the deviation is 0 at $\mathrm{u}=0.5$, which is the point of maximum uncertainty in the interpolant. It is clear a reasonable estimate of the uncertainty of the interpolating curve can be obtained by considering the position and surface normal variation independently.

Having a model for the effect of measurement errors upon the interpolating curve, it is possible to find the magnitude and distribution of the uncertainty of the interpolating curve based upon statistical values for the expected measurement variation of position and surface normal results. The uncertainties from the position measurements combine to produce a rectangular region between the two measurement points of width equal to the total position measurement uncertainty.

The surface normal uncertainties combine to produce the largest deviation when the signs of the normals are opposite. The maximum variation occurs at the midpoint between the samples. The magnitude of the maximum or minimum is to $\pm 0.25 \mathrm{dN}_{\mathrm{u}}$, where $\mathrm{d}$ is the distance between sample points, and $\mathrm{N}_{\mathrm{u}}$ is the standard uncertainty of the surface normal measurement in radians. Figure 3 shows the total combined uncertainty zone for the interpolant. 


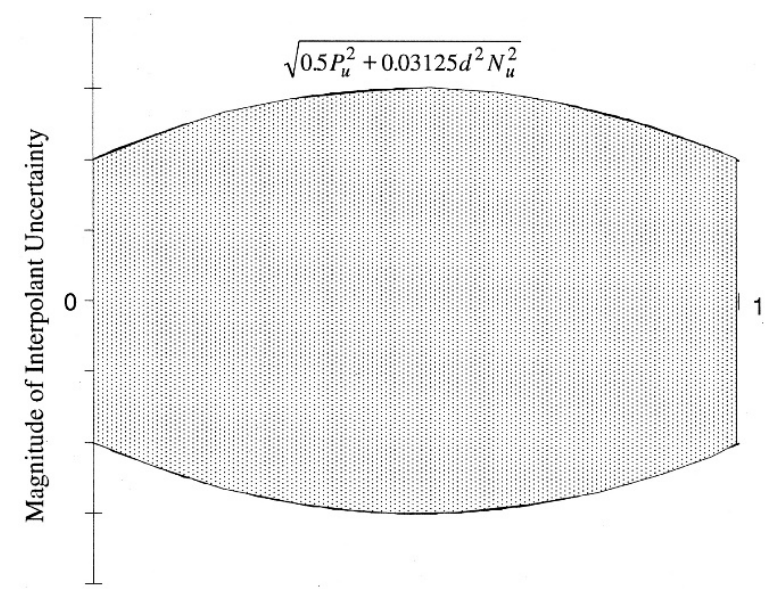

Figure 3. Shape of the combined uncertainty zone for the cubic interpolant.

Representing the standard position measurement uncertainty by $\mathrm{Pu}$ and the surface normal measurement variation by $\mathrm{Nu}$, the maximum combined variation at the midpoint between samples is:

$$
\text { Max Variation } u=0.5= \pm[0.5 \mathrm{Pu}(0)+0.5 \mathrm{Pu}(1)+0.125 \mathrm{dNu}(0)+0.125 \mathrm{dNu}(1)]
$$

The variation computed above is an upper bound for the uncertainty of the interpolant attributable to measurement uncertainty. Because there are four independent parameters contributing to the variation that will combine statistically, it is an inherently pessimistic estimator. Assuming that the measuring uncertainties are independent, the combined uncertainty can be shown to be [Eq. (3)]:

$$
\sqrt{0.5 P_{U}^{2}+0.03125 d^{2} N_{U}^{2}}
$$

Table 1 shows the $95 \%$ confidence zone of the interpolant at various sample separations for two cases. The first case shows the uncertainties for the dataset used as an example later in this text. The expanded position uncertainty for this data set was $\pm 0.002 \mathrm{~mm}$ and the expanded surface normal uncertainty was estimated to be 0.0004 radians. The second set of uncertainty values is based upon the results of an uncertainty analysis of a scanning CMM at a manufacturer's facility. 
Table 1. 95\% Confidence interval on the variation of interpolation curve resulting from measuring uncertainty using combined uncertainties

\begin{tabular}{lcc}
\hline $\begin{array}{l}\text { Surface distance } \\
\text { between samples } \\
(\mathrm{mm})\end{array}$ & $\begin{array}{c}\text { Variation for example data } \\
P u=0.002 \mathrm{~mm}\end{array}$ & $\begin{array}{c}\text { Variation for scanning cmm } \\
P u=0.0018 \mathrm{~mm} \\
N u=8 \text { arc min }\end{array}$ \\
\hline 1 & $N u$ arc min & $\pm 0.0013 \mathrm{~mm}$ \\
10 & $\pm 0.0014 \mathrm{~mm}$ & $\pm 0.0043 \mathrm{~mm}$ \\
25 & $\pm 0.0015 \mathrm{~mm}$ & $\pm 0.010 \mathrm{~mm}$ \\
50 & $\pm 0.0026 \mathrm{~mm}$ & $\pm 0.020 \mathrm{~mm}$ \\
250 & $\pm 0.0038 \mathrm{~mm}$ & $\pm 0.102 \mathrm{~mm}$ \\
\hline
\end{tabular}

Both sets of data suggest that the interpolation model could be useful in a wide range of measuring applications. A measurement system capable of measuring surface inclinations to \pm 1.4 arc seconds, such as shown in the first dataset, could generate error estimates for samples spaced 50-mm apart with uncertainty less than double the point measurement variation.

\subsection{Sampling algorithm}

The interpolation model is used to allow the measuring system to determine a sampling plan autonomously for individual parts that will consistently produce a reliable and complete measurement. The interpolant identifies areas where further samples may be required for a complete measurement. After additional samples are collected, the process is repeated until all completion criteria are satisfied. The process is outlined below, and Figure 4 is a flow chart for the measurement cycle.

1. Initially, sample the profile. Enough samples should be taken so that it is possible to fit the measurement data to the nominal profile. The initial sample points should also include the endpoints of the region of interest.

2. Fit the measurement data to the nominal geometry using an appropriate algorithm. This step is necessary to ensure that the measurement points are correlated to point on the nominal surface with minimal error.

3. Calculate the position and surface normal errors for each point as described above. Use these data to develop an interpolation curve between each pair of points.

4. Evaluate each piece of the interpolating spline for new target points, and sample the new targets. The criteria for selecting new target points are discussed below.

5. Return to step 2, and repeat the process until no new target points are located. 


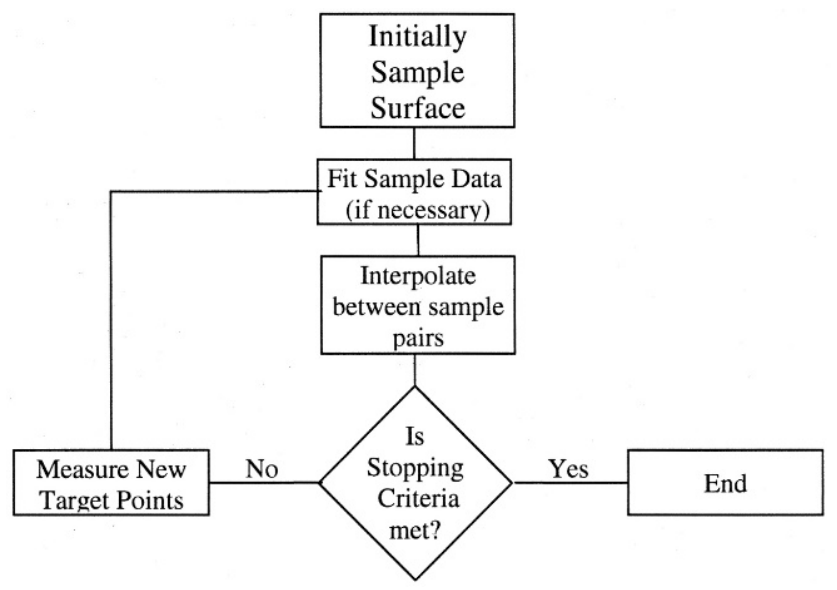

Figure 4. Flow chart of sampling algorithm.

Step 4 requires that the algorithm be able to determine the nominal position and surface normal for any point on the surface profile. This could be achieved by either maintaining a mathematical model of the surface in the CMM controller or by supplying a file with a dense array of nominal coordinates and surface normals from which the program could select the closest match to the ideal target. We should note that, for general application, the CMM must have the ability to find a collision-free path from its current position to the new targets. Although it is beyond the scope of this paper, the authors are satisfied that this could be achieved by reverse-engineering the CMM's original path across the profile.

Depending upon the size of the surface being measured, the processing speed for fitting, and the maximum drive speed of the CMM, it may be desirable to iterate to the stop criteria between pairs of points instead of searching the entire surface. This approach would minimize the distance traveled by the CMM.

\subsection{Criteria for selecting new target points}

Although the ideal set of criteria for choosing new target points has not been determined, presently we are using three general rules to decide when and where further samples are necessary. These are as follows.

1. Sample at the extreme location(s) on the interpolating curve if the error curve exceeds the bounding box formed by the sample points plus an offset value.

2. If the interpolation curve does not violate the sampling boundaries described above, but the distance between sample points is greater than the maximum spacing between sample points, probe at the midpoint between the pair of samples.

3. If the spacing between sample points is less than or equal to the minimum spacing between sample points parameter, do not sample further, regardless of the previous two rules.

Other rules could be implemented, depending upon the measurement application. For example, a step between sample points will produce an inflection point in the interpolation 
curve. If it desirable to locate the location of steps, it would be necessary to sample at inflection points.

\subsection{Limitations of the search algorithm}

The algorithm we have described depends upon the assumption that the manufacturing errors are continuous. One source of discontinuous errors is the surface roughness of the part. Discontinuities could also be the result of errors in the process plan or one-time events specific to only a single part from a manufacturing run.

The effect of surface finish upon the algorithm is difficult to characterize precisely. As the surfaces being measured become rougher, an effective irreproducibility will result, because probing two closely spaced points could result in a discontinuity of surface normal measurements. Efforts to define the interaction of surface finish and surface normal measurements better are currently underway.

Process planning errors that result in discontinuities, such as burrs or gouges, would be difficult to detect consistently using our search algorithms. However, these types of errors should be designed out of the process at the prototyping stage and would not normally appear during a production run.

As with any iterative method, the final result is sensitive to the starting conditions of the algorithm. The number of measurements required for convergence will vary, depending upon the location of the initial sample points.

\subsection{Simulated testing of the search algorithm}

Several test cases have been implemented to confirm the utility of the sampling algorithm, all with positive results. Examples of simple bending, sinusoidal error profiles, and combinations of trigonometric functions all converge quickly and reliably to a complete and accurate representation of the true error profile.

The test case presented here uses actual measurement data collected as part of a study to determine the correlation of part errors with standard machine tool performance tests [21]. The data consisted of 360 measurements of a $300-\mathrm{mm}$ diameter circular contour cut on a horizontal machining center. Figure 5 shows a roundness plot of the CMM data. The dataset contains large errors at 0 and $180^{\circ}$ because of backlash in the $x$-axis. The very large, sharp discontinuity at $270^{\circ}$ is the result of a velocity error when the machining process changes from a drilling operation to a contouring operation at the beginning of the cut. This dataset presents a significant challenge to any sampling plan because of the large amount of process data available, as well the sharp discontinuity at $270^{\circ}$. 


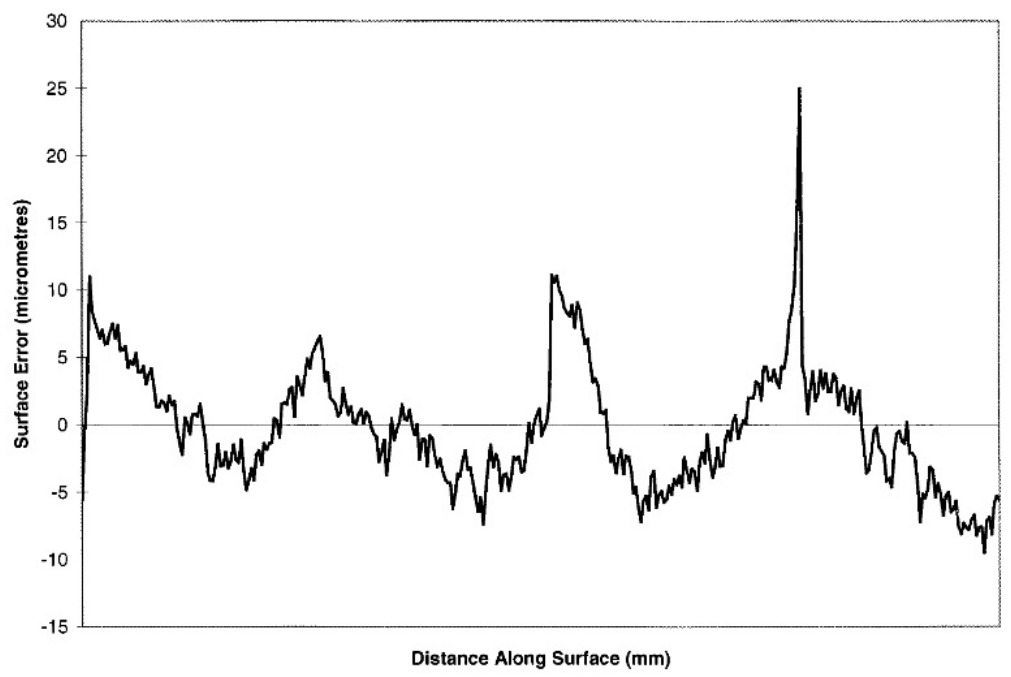

Figure 5. Contouring error data used for simulated testing of the search algorithm.

The data were collected using a point probing CMM in an environment controlled to 20 $\pm 0.1^{\circ} \mathrm{C}$. Because surface normal data was unavailable, it was estimated by numerically differentiating the measured deviations from the nominal circle. The appendix contains the complete set of surface normal and position deviations used in the experiment.

An initial sample size of three points was selected to allow a circle fit to be computed. The samples were uniformly distributed. If process knowledge were used, the algorithm could be optimized by starting the process at one of the coordinate axes. To make our test as realistic as possible, we choose to forego this advantage and take our initial data equally spaced starting at $20^{\circ}$ from the $x$-axis. The threshold for choosing to sample a predicted extrema was set to $2 \mathrm{~mm}$. The minimum separation between sample points was $2^{\circ}$, and the maximum separation between sample points was $60^{\circ}$. The algorithm described above was applied and terminated after 27 points were collected in total. Figure 6 shows the graph of position error as measured by the CMM and as represented by the cubic spline model after completion of the search algorithm. A uniformly spaced sampling strategy would require more than six times as many points to achieve the same minimum feature resolution. 


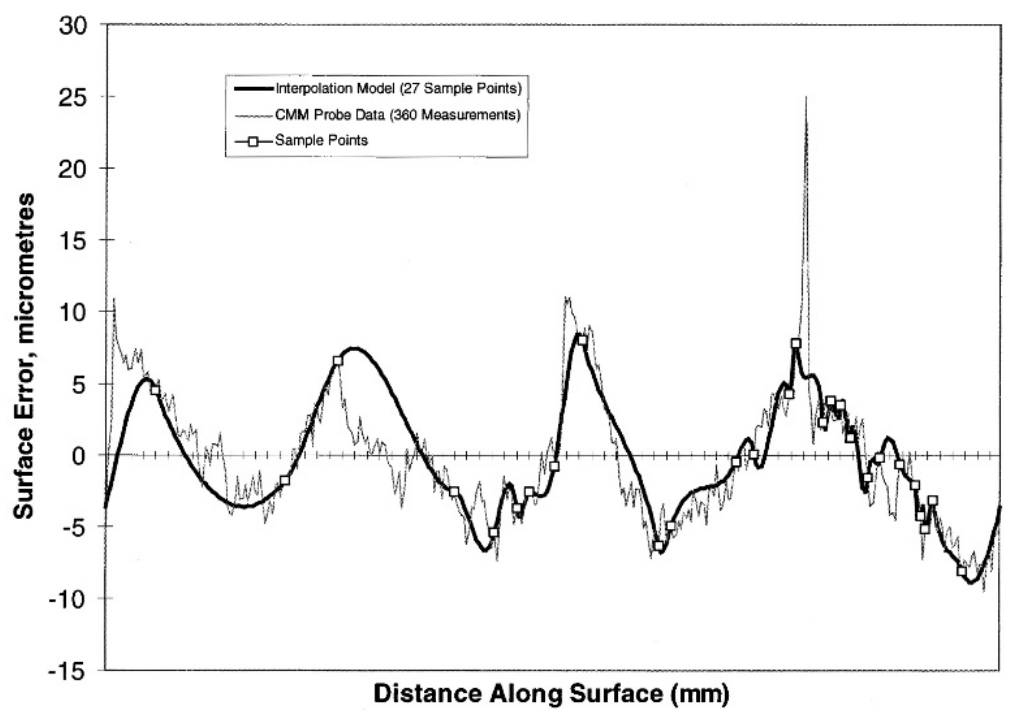

Figure 6. Performance of sampling algorithm.

As can be seen from the figure, the algorithm results in a good estimate of the shape of the surface. If we exclude the discontinuity at $270^{\circ}$, the profile error found by the 360 point measurement is $20.6 \mu \mathrm{m}$, the iterative method finds a profile error of $17.3 \mu \mathrm{m}$. The discrepancy in the two results is attributable to the discontinuous nature of the backlash error at 0 and $180^{\circ}$. Although the exact magnitude of the backlash errors is not identified, a large error is clearly indicated in these areas.

\section{Conclusions}

An innovative technique has been developed for the adaptive sampling of part surfaces measured with a coordinate measuring system. The technique is predicated on a sample of position $\langle\mathrm{x}, \mathrm{y}, \mathrm{z}>$ and surface normal $<\mathrm{i}, \mathrm{j}, \mathrm{k}>$ at each measurement point. Using this extended dataset and a description of the nominal geometry for a measured part, it is shown that an estimate of the error surface can be made incrementally as sample points are acquired.

Each new estimate of the error surface is used to choose the next sample point on the part surface. The error surface is developed using a cubic spline representation. The diminishing variation properties of this model ensure that the error model will always have fewer or an equal number of spatial undulations, as compared to the actual part surface. As the sample size increases, the error surface converges to match the error of the actual surface closely. Current stopping criteria include comparison of tolerance to estimated error and comparison of spatial frequency of the error surface to expected spatial frequency for the part. The data and computation requirements for the technique are quite small. The technique is always conservative in estimating the error surface. When the error surface differs from the actual part surface, the maximum estimated error always exceeds the error of the actual part surface. Uncertainty analysis is developed to show how the uncertainty 
of the estimated error surface is related to the magnitude and frequency of measurement errors. Example data from a high-precision part and a high-precision measurement instrument suggest that the uncertainty associated with this technique can be controlled and compares well with the uncertainty of the measuring instrument.

For an equal number of samples, the method will require more measurement time than a uniform sampling pattern, because of the extra drive moves required by the algorithm. However, because the algorithm generates sample points concentrated in the areas of large error, the number of sample points required will be consistently less than that required by a uniform pattern for an equally complete measurement. Note that the sampling strategy described in this paper will automatically sample sparsely on acceptable parts or parts exhibiting high process capability, while requiring that extensive samples be made on parts of low process capability. The process will perform particularly well in comparison to a static process sufficient to measure poor quality parts completely, when high-quality parts are inspected.

The technique has been described and demonstrated for 2-D problems and has immediate practical applications. A three-dimensional (3-D) extension is currently being developed. The algorithm can also be immediately applied to the problem of identifying outliers in scanned surface data. The error surface estimate would be incrementally built up from the data points acquired by a scanning coordinate metrology probe. Outliers would be identified by considering deviations at surface points with respect to the nominal curvature of the part surface and the maximum spatial frequency expected for the part surface.

Acknowledgments - We thank Dr. Stuart Smith and his students for providing insights into the measurement of surface normals. W also thank Cummins Engine Company for providing data used in our study. This work has benefited greatly from the exceptional research environment provided by the Center for Precision Metrology at UNC Charlotte and the Cameron Center for Applied Research. This material is based upon work supported by the National Science Foundation under Grant No. DMI-9457168. 
EdgeWorth AND Wilhelm, PRECISION ENGINEERING 23 (1999)

\section{Appendix}

\begin{tabular}{|c|c|c|c|c|c|c|c|c|}
\hline \multicolumn{9}{|c|}{ Test data and sample calculation of interpolating curve } \\
\hline Angle & $\varepsilon \mu \mathrm{m}$ & $\varepsilon^{\prime} \times 10^{-3}$ & Angle & $\varepsilon \mu \mathrm{m}$ & $\varepsilon^{\prime} \times 10^{-3}$ & Angle & $\varepsilon \mu \mathrm{m}$ & $\varepsilon^{\prime} \times 10^{-3}$ \\
\hline 0 & -5.6 & 1.19 & 45 & 0.6 & 0.25 & 90 & 5.3 & 0.36 \\
\hline 1 & -0.4 & 1.35 & 46 & 1.5 & -0.04 & 91 & 5.7 & 0.13 \\
\hline 2 & 2.2 & 2.53 & 47 & 0.1 & -0.56 & 92 & 6.2 & 0.24 \\
\hline 3 & 11.0 & 1.40 & 48 & -1.1 & -0.77 & 93 & 6.6 & -0.11 \\
\hline 4 & 8.4 & -1.18 & 49 & -3.6 & -0.64 & 94 & 5.4 & -0.83 \\
\hline 5 & 7.7 & -0.09 & 50 & -4.1 & -0.07 & 95 & 3.3 & -0.20 \\
\hline 6 & 7.1 & -0.31 & 51 & -4.2 & 0.17 & 96 & 3.9 & -0.20 \\
\hline 7 & 6.4 & 0.06 & 52 & -3.1 & 0.72 & 97 & 2.0 & -0.53 \\
\hline 8 & 7.0 & -0.07 & 53 & -1.5 & -0.06 & 98 & 1.8 & 0.02 \\
\hline 9 & 6.0 & -0.29 & 54 & -3.1 & -0.49 & 99 & 1.5 & -0.27 \\
\hline 10 & 6.1 & 0.24 & 55 & -3.0 & 0.39 & 100 & 0.7 & -0.21 \\
\hline 11 & 6.9 & 0.37 & 56 & -2.1 & -0.08 & 101 & 1.0 & 0.57 \\
\hline 12 & 7.5 & -0.21 & 57 & -3.2 & -0.27 & 102 & 2.7 & 0.16 \\
\hline 13 & 6.4 & 0.05 & 58 & -2.7 & 0.52 & 103 & 1.5 & -0.58 \\
\hline 14 & 7.4 & -0.16 & 59 & -1.5 & 0.01 & 104 & 0.8 & 0.08 \\
\hline 15 & 5.5 & -0.50 & 60 & -2.6 & -0.46 & 105 & 1.3 & -0.10 \\
\hline 16 & 5.6 & 0.26 & 61 & -2.8 & 0.52 & 106 & 0.2 & -0.37 \\
\hline 17 & 5.8 & -0.35 & 62 & -1.1 & -0.06 & 107 & 0.0 & 0.17 \\
\hline 18 & 4.3 & -0.26 & 63 & -3.4 & -0.98 & 108 & 0.8 & 0.31 \\
\hline 19 & 4.7 & 0.08 & 64 & -4.8 & -0.11 & 109 & 1.1 & -0.23 \\
\hline 20 & 4.5 & 0.17 & 65 & -4.2 & 0.48 & 110 & 0.1 & -0.06 \\
\hline 21 & 5.3 & -0.11 & 66 & -3.3 & -0.12 & 111 & 0.9 & 0.21 \\
\hline 22 & 3.9 & -0.41 & 67 & -4.1 & 0.18 & 112 & 0.6 & -0.30 \\
\hline 23 & 3.9 & 0.24 & 68 & -2.2 & 0.62 & 113 & -0.2 & -0.29 \\
\hline 24 & 4.3 & -0.23 & 69 & -1.9 & -0.36 & 114 & -0.7 & -0.01 \\
\hline 25 & 3.0 & -0.21 & 70 & -3.0 & 0.14 & 115 & -0.9 & -0.49 \\
\hline 26 & 3.6 & 0.39 & 71 & -1.4 & 0.31 & 116 & -2.7 & -0.35 \\
\hline 27 & 4.2 & -0.14 & 72 & -1.8 & -0.08 & 117 & -2.1 & 0.60 \\
\hline 28 & 2.9 & -0.68 & 73 & -1.4 & 0.06 & 118 & -1.2 & -0.49 \\
\hline 29 & 1.4 & -0.33 & 74 & -1.3 & 0.41 & 119 & -3.7 & -0.44 \\
\hline 30 & 1.3 & 0.17 & 75 & 0.4 & 0.47 & 120 & -2.2 & 1.21 \\
\hline 31 & 1.8 & 0.10 & 76 & 0.3 & -0.54 & 121 & 0.4 & 0.13 \\
\hline 32 & 1.6 & -0.26 & 77 & -1.0 & 0.29 & 122 & -1.1 & -0.33 \\
\hline 33 & 1.0 & 0.17 & 78 & 1.5 & 0.69 & 123 & -0.3 & 0.32 \\
\hline 34 & 2.1 & 0.11 & 79 & 1.6 & -0.20 & 124 & 0.2 & 0.43 \\
\hline 35 & 1.5 & -0.05 & 80 & 1.5 & 0.22 & 125 & 1.5 & 0.05 \\
\hline 36 & 1.7 & -0.34 & 81 & 2.6 & 0.43 & 126 & 0.4 & -0.38 \\
\hline 37 & -0.3 & -0.69 & 82 & 2.8 & -0.68 & 127 & 0.3 & 0.29 \\
\hline 38 & -1.4 & -0.48 & 83 & 0.6 & 0.21 & 128 & 1.1 & -0.07 \\
\hline 39 & -2.2 & 0.50 & 84 & 3.6 & 0.72 & 129 & -0.1 & -0.53 \\
\hline 40 & 0.5 & 0.64 & 85 & 3.0 & -0.53 & 130 & -0.7 & 0.24 \\
\hline 41 & 0.1 & -0.50 & 86 & 2.2 & 0.09 & 131 & 0.0 & -0.48 \\
\hline 42 & -0.7 & 0.18 & 87 & 3.5 & 0.71 & 132 & -2.5 & -0.31 \\
\hline 43 & 0.7 & 0.40 & 88 & 4.9 & 0.06 & 133 & -1.1 & 0.60 \\
\hline 44 & 0.8 & -0.17 & 89 & 4.2 & 0.01 & 134 & -1.1 & -0.67 \\
\hline
\end{tabular}

(continued on next page) 
EdgeWorth ANd Wilhelm, PRECISION ENGINEERING 23 (1999)

\begin{tabular}{|c|c|c|c|c|c|c|c|c|}
\hline \multicolumn{9}{|c|}{ Appendix (continued) } \\
\hline Angle & $\varepsilon \mu \mathrm{m}$ & $\varepsilon^{\prime} \times 10^{-3}$ & Angle & $\varepsilon \mu \mathrm{m}$ & $\varepsilon^{\prime} \times 10^{-3}$ & Angle & $\varepsilon \mu \mathrm{m}$ & $\varepsilon^{\prime} \times 10^{-3}$ \\
\hline 135 & -3.0 & 0.08 & 180 & -0.8 & -0.36 & 225 & -6.1 & -0.46 \\
\hline 136 & -0.8 & 0.63 & 181 & -0.2 & 0.30 & 226 & -5.2 & 0.47 \\
\hline 137 & -1.0 & -0.42 & 182 & 0.2 & -0.03 & 227 & -4.9 & -0.19 \\
\hline 138 & -2.2 & -0.49 & 183 & 1.9 & 2.60 & 228 & -5.8 & -0.21 \\
\hline 139 & -3.1 & 0.02 & 184 & 11.1 & 1.94 & 229 & -5.5 & 0.37 \\
\hline 140 & -2.5 & 0.01 & 185 & 10.6 & -0.43 & 230 & -4.6 & 0.02 \\
\hline 141 & -3.3 & -0.37 & 186 & 11.0 & -0.04 & 231 & -5.1 & 0.10 \\
\hline 142 & -4.0 & -0.19 & 187 & 10.0 & -0.33 & 232 & -4.0 & 0.17 \\
\hline 143 & -4.3 & 0.08 & 188 & 9.6 & -0.24 & 233 & -4.4 & 0.06 \\
\hline 144 & -4.3 & -0.50 & 189 & 8.7 & -0.27 & 234 & -3.8 & -0.14 \\
\hline 145 & -6.2 & -0.26 & 190 & 8.3 & -0.15 & 235 & -4.6 & 0.33 \\
\hline 146 & -5.1 & 0.73 & 191 & 8.1 & 0.24 & 236 & -2.4 & 0.38 \\
\hline 147 & -3.6 & 0.18 & 192 & 8.9 & -0.28 & 237 & -3.4 & -0.61 \\
\hline 153 & -4.3 & -0.49 & 198 & 6.3 & -0.20 & 243 & -2.1 & -0.28 \\
\hline 154 & -5.4 & -0.51 & 199 & 4.7 & -0.78 & 244 & -2.9 & 0.39 \\
\hline 155 & -6.4 & 0.18 & 200 & 3.2 & -0.20 & 245 & -0.7 & 0.19 \\
\hline 156 & -5.4 & -0.28 & 201 & 3.4 & 0.10 & 346 & -2.5 & -0.89 \\
\hline 157 & -7.4 & -0.07 & 202 & 2.9 & -0.60 & 247 & -3.9 & -0.12 \\
\hline 158 & -5.0 & 1.09 & 203 & 0.9 & -0.48 & 248 & -3.2 & 0.63 \\
\hline 159 & -2.8 & 0.78 & 204 & 0.8 & 0.28 & 249 & -1.7 & -0.02 \\
\hline 160 & -1.5 & -0.21 & 205 & 1.1 & -0.54 & 250 & -3.1 & -0.46 \\
\hline 161 & -3.1 & -0.20 & 206 & -1.6 & -0.94 & 251 & -3.0 & 0.49 \\
\hline 162 & -2.2 & 0.28 & 207 & -2.7 & 0.06 & 252 & -1.2 & 0.63 \\
\hline 163 & -2.8 & -0.75 & 208 & -2.3 & -0.21 & 253 & -0.5 & -0.15 \\
\hline 164 & -4.9 & -0.19 & 209 & -3.6 & -0.13 & 254 & -1.1 & 0.11 \\
\hline 165 & -3.7 & 0.46 & 210 & -2.5 & 0.56 & 255 & 0.2 & 0.56 \\
\hline 166 & -3.6 & -0.38 & 211 & -1.9 & -0.39 & 256 & 0.7 & -0.40 \\
\hline 167 & -4.8 & -0.12 & 212 & -3.6 & -0.13 & 257 & -1.0 & -0.29 \\
\hline 168 & -3.8 & 0.64 & 213 & -2.3 & 0.42 & 258 & -0.3 & 0.43 \\
\hline 169 & -2.4 & 0.22 & 214 & -2.4 & -0.19 & 259 & 0.3 & -0.05 \\
\hline 170 & -2.6 & 0.00 & 215 & -3.2 & -0.66 & 260 & 0.1 & 0.36 \\
\hline 171 & -2.4 & -0.19 & 216 & -5.1 & -0.22 & 261 & 2.0 & 0.48 \\
\hline 172 & -3.5 & -0.32 & 217 & -4.6 & -0.11 & 262 & 2.0 & -0.17 \\
\hline 173 & -3.4 & 0.36 & 218 & -6.2 & -0.71 & 263 & 2.0 & 0.29 \\
\hline 174 & -1.8 & 0.88 & 219 & -7.2 & 0.20 & 264 & 3.2 & 0.33 \\
\hline 175 & 0.1 & -0.03 & 220 & -5.6 & 0.56 & 265 & 3.1 & -0.51 \\
\hline 176 & -1.3 & -0.13 & 221 & -5.3 & -0.38 & 266 & 1.9 & 0.30 \\
\hline 177 & 0.0 & 0.51 & 222 & -6.3 & 0.29 & 267 & 4.3 & 0.69 \\
\hline 178 & 0.7 & 0.30 & 223 & -3.8 & 0.88 & 268 & 4.3 & -0.36 \\
\hline 179 & 1.2 & -0.40 & 224 & -3.4 & -0.71 & 269 & 3.3 & -0.27 \\
\hline
\end{tabular}

(continued on next page) 
EdgeWORTH ANd WiLHELM, PRECISION ENGINEERING 23 (1999)

\begin{tabular}{|c|c|c|c|c|c|c|c|c|}
\hline \multicolumn{9}{|c|}{ Appendix (continued) } \\
\hline Angle & $\varepsilon \mu \mathrm{m}$ & $\varepsilon^{\prime} \times 10^{-3}$ & Angle & $\varepsilon \mu \mathrm{m}$ & $\varepsilon^{\prime} \times 10^{-3}$ & Angle & $\varepsilon \mu \mathrm{m}$ & $\varepsilon^{\prime} \times 10^{-3}$ \\
\hline 270 & 3.3 & 0.28 & 300 & 1.0 & 0.53 & 330 & -5.4 & -0.13 \\
\hline 271 & 4.1 & 0.00 & 301 & 2.7 & -0.08 & 331 & -4.8 & 0.57 \\
\hline 272 & 3.2 & -0.44 & 302 & 0.8 & -0.31 & 332 & -3.1 & 0.42 \\
\hline 273 & 2.7 & 0.33 & 303 & 1.9 & 0.64 & 333 & -3.4 & -0.67 \\
\hline 274 & 4.3 & 0.31 & 304 & 2.5 & -0.49 & 334 & -5.4 & -0.19 \\
\hline 275 & 4.2 & 0.07 & 305 & -0.1 & 0.89 & 335 & -4.4 & 0.25 \\
\hline 276 & 5.5 & 0.83 & 306 & -1.6 & -0.67 & 336 & -5.1 & -0.68 \\
\hline 277 & 7.8 & 0.58 & 307 & -3.6 & -0.41 & 337 & -6.7 & -0.03 \\
\hline 278 & 8.5 & 0.30 & 308 & -3.3 & 0.39 & 338 & -5.3 & 0.55 \\
\hline 279 & 10.5 & 1.26 & 309 & -2.0 & 0.64 & 339 & -5.0 & -0.36 \\
\hline 280 & 16.3 & 4.34 & 310 & -0.4 & 0.42 & 340 & -6.5 & -0.34 \\
\hline 281 & 25.0 & -3.04 & 311 & -0.2 & -0.35 & 341 & -6.3 & 0.36 \\
\hline 282 & 4.5 & -5.35 & 312 & -1.7 & -0.40 & 342 & -5.7 & -0.29 \\
\hline 283 & 3.4 & 0.16 & 313 & -1.9 & 0.02 & 343 & -7.6 & -0.63 \\
\hline 284 & 0.8 & -0.36 & 314 & -2.3 & -0.54 & 344 & -8.1 & 0.17 \\
\hline 285 & 2.6 & 0.97 & 315 & -4.2 & -0.34 & 345 & -7.3 & 0.13 \\
\hline 286 & 3.9 & -0.30 & 316 & -4.0 & -0.13 & 346 & -7.6 & -0.18 \\
\hline 287 & 1.8 & -0.55 & 317 & -4.6 & 0.40 & 347 & -7.8 & 0.13 \\
\hline 288 & 2.3 & 0.72 & 318 & -1.9 & 0.95 & 348 & -7.1 & 0.33 \\
\hline 289 & 4.0 & 0.00 & 319 & -0.6 & 0.23 & 349 & -6.7 & -0.34 \\
\hline 290 & 2.7 & -0.07 & 320 & -0.5 & -0.17 & 350 & -8.2 & -0.24 \\
\hline 291 & 3.8 & 0.02 & 321 & -1.2 & -0.30 & 351 & -7.6 & 0.34 \\
\hline 292 & 2.4 & -0.45 & 322 & -1.4 & 0.48 & 352 & -7.6 & -0.59 \\
\hline 293 & -2.5 & 0.38 & 323 & 0.2 & -0.17 & 353 & -9.5 & 0.09 \\
\hline 294 & 3.7 & 0.34 & 324 & -2.1 & -0.62 & 354 & -7.1 & 0.78 \\
\hline 295 & 3.5 & -0.65 & 325 & -2.1 & 0.13 & 355 & -6.9 & -0.48 \\
\hline 296 & 1.5 & -0.25 & 326 & -2.4 & -0.35 & 356 & -8.1 & 0.23 \\
\hline 297 & 2.5 & 0.52 & 327 & -4.2 & -1.23 & 357 & -5.8 & 0.74 \\
\hline 298 & 2.9 & -0.34 & 328 & -7.2 & -0.10 & 358 & -5.3 & -0.06 \\
\hline 299 & 1.2 & -0.57 & 329 & -5.1 & 0.54 & 359 & -5.6 & -0.32 \\
\hline
\end{tabular}

We want to find the cubic curve that interpolates between the sample points at 3 and $25^{\circ}$. The Hermite form of the solution presumes a curve with endpoints at $u=0$ and $u=1$. We need to scale the derivatives by the distance between the sample points to correct for this parametrization. The spacing between sample points for this dataset is $2.6 \mathrm{~mm}$. Maintaining units of micrometers throughout, we multiply both error derivatives by $57.2 \times 10^{3}$. The cubic coefficients can then be found using:

$$
\begin{array}{llr}
\mathrm{a}_{0}=\varepsilon(3) & = & 11.0 \\
\mathrm{a}_{1}=\left(57.2 \times 10^{3}\right) \varepsilon^{\prime}(3) & = & 80.1 \\
\mathrm{a}_{2}=-3 \varepsilon(3)+3 \varepsilon(25)-2\left(57.2 \times 10^{3}\right) \varepsilon^{\prime}(3)-\left(57.2 \times 10^{3}\right) \varepsilon^{\prime}(25) & = & -172.1 \\
a_{3}=2 \varepsilon(3)-2 \varepsilon(25)+\left(57.2 \times 10^{3}\right) \varepsilon^{\prime}(3)+\left(57.2 \times 10^{3}\right) \varepsilon^{\prime}(1) & = & 84.1
\end{array}
$$

So,

$$
\varepsilon(u)=11.0+80.1 u-172.1 u^{2}+84.1 u^{3} \quad 0 \leq u \leq 1
$$

is the interpolating curve between the sample points at 3 and $25^{\circ}$. 


\section{References}

[1] Summerhays KD, Henke RP, Cassou RM, Baldwin JM, Brown CW. New algorithms for the evaluation of discrete point measurement data and for sample point selection on surfaces with systematic form deviations. Proc ASPE 1995; 12: 388-91.

[2] Mestre M, Abou-Kandil H. Measuring the errors of form of industrial surfaces: Prediction and optimization. Prec Eng 1994; 16: 268-75.

[3] Woo TC, Liang R. Dimensional measurement of surfaces and their sampling. Comp Aided Design 1993; 25: 233-9.

[4] Liang R, Woo TC, Hsieh C. Accuracy and time in surface measurement, Part 1: Mathematical foundations. J Manufact Sci Eng 1998; 120: 141-49.

[5] Liang R, Woo TC, Hsieh C. Accuracy and time in surface measurement, Part 2: Optimal sampling sequence. J Manufact Sci Eng 1998; 120: 50-155.

[6] Edgeworth R, Wilhelm RG. Substitute geometry fitted from surface normal information. Proc Am Soc Prec Eng 1996; 14: 229-32.

[7] Aoyama H, Kawai M, Kishinami T. A new method for detecting the contact point between a touch probe and a surface. Ann CIRP 1989; 38: 593-96.

[8] Yamazaki K, Aoyama H, Lee KS, Sawabe M. Noncontact probe for continuous measurement of surface inclination and position using dynamic irradiation of light beam. Ann CIRP 1993; 42: 585-88.

[9] Vidic M, Harb SM, Smith ST. Observations of contact measurements using a resonance-based touch sensor. Proc Am Soc Prec Eng 1996; 14: 41-50.

[10] Vidic M, Harb SM, Smith ST. Observations of contact measurements using a resonance-based touch sensor. Prec Eng 1998; 22: 19-36.

[11] Hocken RJ, Raja J, Babu U. Sampling issues in coordinate metrology. Manufact Rev 1993; 6: $282-94$.

[12] Kim N, Kim S. Geometrical tolerances: Improved linear approximation of least-squares evaluation of circularity by minimum variance. Int J Mach Tools Manufact 1996; 36: 355-66.

[13] Cheragi S, Huay S, Motavalli S. Straightness and flatness tolerance evaluation: An optimization approach. Prec Eng 1996; 18: 30-37.

[14] Carr K, Ferriera P. Verification of form tolerance, Part I: Basic issues, flatness, and straightness. Prec Eng 1995; 17: 131-43.

[15] Carr K, Ferriera P. Verification of form tolerance, Part II: Cylindricity and straightness of a median line. Prec Eng 1995; 17: 144-56.

[16] Omitted.

[17] Menq C-H, Yau H-T, Lai G-W. Automated precision measurement of surface profile in CAD directed inspection. IEEE Trans Robot Automat 1992; 8: 268-78.

[18] Duffie N, Bollinger J, Piper R, Kroneberg M. CAD directed inspection and error analysis using surface patch databases. Ann CIRP 1984; 33: 347-50.

[19] Weckenmann A, Eitzert H, Garmer M, Weber H. Functionality oriented evaluation and sampling strategy in coordinate metrology. Prec Eng 1995; 17: 244-52.

[20] Farin G. Curves and surfaces for computer-aided geometric design: A practical guide (3rd ed.). San Diego: Academic Press, 1993.

[21] Wilhelm RG, Srinivasan N, Farabaugh F, Hocken R. Part form errors predicted from machine tool performance measurements. Ann CIRP 1997; 46: 471-74. 